\title{
Structure-activity relationship of a series of antioxidant tripeptides derived from $\beta$-Lactoglobulin using QSAR modeling
}

\author{
Mai Tian ${ }^{1} \cdot$ Bing Fang $^{2} \cdot$ Lu Jiang $^{1}$. \\ Huiyuan $\mathrm{Guo}^{3} \cdot \mathrm{JianYun} \mathrm{Cui}^{4} \cdot$ Fazheng Ren ${ }^{1}$
}

Received: 30 September 2014 / Revised: 16 March 2015 / Accepted: 23 March 2015 /

Published online: 7 May 2015

(C) INRA and Springer-Verlag France 2015

\begin{abstract}
Antioxidative peptides derived from food proteins have been screened and characterized in in vitro assays, and several quantitative structure-activity relationship (QSAR) models have been established to understand the structure-activity relationship of the selected antioxidant peptides. However, a systematic evaluation of each fragment that could be produced by a certain protein is still lacking. In this study, all the tripeptides that could possibly derive from bovine beta-lactoglobulin based on its amino acid sequence have been synthesized, and their antioxidant activities were measured using a ferric-reducing antioxidant power assay. Three of the peptides (i.e., LTC, CQC, and GTW) exhibited higher activities than that of glutathione. A QSAR model was also computed using multiple linear regression with divided physicochemical property scores descriptors, and the validity of the model was confirmed by cross-validation and Y-scrambling. The result of the QSAR modeling indicated that the electronic and hydrogen-bonding properties of the amino acids in the tripeptide sequences, as well as the steric properties of the amino acid residues at the $\mathrm{C}$ - and $\mathrm{N}$-termini, played an important role in the antioxidant activities of the tripeptides. The antioxidant activities of the tripeptides were generally higher with Cys and Trp amino acid residues in the sequence. The structural characteristics we found may contribute to the further research of antioxidative mechanism.
\end{abstract}

Fazheng Ren

renfazheng@263.net

1 Key Laboratory of Functional Dairy, College of Food Science and Nutritional Engineering, China Agricultural University, Beijing 100083, China

2 Academy of State Administration of Grain, Beijing 100037, China

3 Beijing Key Laboratory of Nutrition, Health \& Food Safety, Beijing 100083, China

4 Beijing Higher Institution Engineering Research Center of Animal Product, Beijing 100083, China 
Keywords QSAR modeling · Tripeptide library - Antioxidant activity· $\beta$-Lactoglobulin • Chemical synthesis

\section{Introduction}

Bioactive peptides are usually 3-20 amino acids in length and are normally inactive within the sequence of the parent animal or plant protein from which they are derived. However, despite their apparent lack of activity, bioactive peptides can exert a variety of different physiological functions after they have been released from the parent protein as a consequence of food processing or enzymatic proteolysis in the gastrointestinal tract (Moller et al. 2008). In contrast to pharmaceutical and chemical additives, protein-derived bioactive peptides exert very few side effects in humans, and there is also a growing preference among consumers for products derived from natural sources (Sakai et al. 1997; Moller et al. 2008). For this reason, considerable research efforts have been focused on the identification and characterization of bioactive peptides derived from various dietary proteins, such as milk, soy, fish, and egg (Samaranayaka and Li-Chan 2011).

$\beta$-Lactoglobulin $(\beta-\mathrm{Lg})$ is a major whey protein that is considered to be an important source of biologically active peptides (Korhonen 2009). Bioactive peptides derived from $\beta$-Lg may function as antihypertensives, antioxidants, and antimicrobials, and can also be used to lower cholesterol (HernandezLedesma et al. 2008). As for antioxidant activity, Liu et al. (2007) found that the antioxidant activity of bovine milk was reduced by $50 \%$ when $\beta-\mathrm{Lg}$ in the milk was depleted. Furthermore, the antioxidant activity of the hydrolysates derived from $\beta-\mathrm{Lg}$ was higher than that of butylated hydroxyanisole, which is the most commonly used antioxidant (Hernández-Ledesma et al. 2005). Therefore, $\beta-\mathrm{Lg}$ is a good template to investigate the structure-activity relationship of antioxidant peptides.

To prepare and isolate protein-derived antioxidant peptides, considerable research efforts have been made towards the optimization of the experimental variables involved in hydrolysis of various dietary proteins, and the following separation and purification of peptides with high levels of activity ( $\mathrm{Gu}$ and $\mathrm{Wu}$ 2013). Despite progress in these areas, the isolation of bioactive peptides using these methods can be tedious, and it is hard to know beforehand the structure or activities of the peptides resulting from hydrolysis. To prepare peptides with high activities more effectively, one possible solution would be to develop a deeper understanding of the relationship between the activity of the bioactive peptides and their structure, which could be used to predict activities of any given structure and instruct whether the parent protein have the potential of preparing highly active peptides.

To identify relationships between the structural features of molecules and their activities, a mathematical method, quantitative structure-activity relationship (QSAR) modeling has been introduced. It provides a method to find mathematical expressions for the relationship between structure and activity, 
which may be useful for estimating or predicting highly active structures. It has been used extensively in this context in the fields of medical science and toxicology. With regard to bioactive peptides, QSAR has been successfully applied to ACE-inhibitory peptides ( $\mathrm{Gu}$ and $\mathrm{Wu}$ 2013; Jing et al. 2014; Wu et al. 2006a, 2006b) and antimicrobial peptides (Jenssen et al. 2008; Yan et al. 2013).

The successful development of QSAR models has been reported to evaluate the antioxidant properties of peptides using the trolox-equivalent antioxidant capacity (TEAC), oxygen radical absorption capacity (ORAC), superoxide radical (SOR), and ferric thiocyanate (FTC) methods (Li and Li 2013; Li et al. 2011). Since the databases of these QSAR models were collected from different sources, there still lacks a systemic evaluation of each fragment that could be produced by a certain protein. Chemically synthesized peptides prepared with well-developed solid-phase synthesis technology provide an alternative solution to obtaining large amounts of peptides with known sequences in a relatively short time, which allows the fast screening of a large amount of sequences for potential biological activities as the database of QSAR modeling. Tripeptides have been reported to exhibit higher levels of antioxidant activity than other oligopeptides (Chen et al. 1996) and are not limited in the same way as dipeptides in terms of the diversity of their structural variations (Jian-Hui et al. 2013). Furthermore, the well-known multifunctional antioxidant, glutathione $(\mathrm{GSH})$ is also a tripeptide composed of sequential Glu, Cys, and Gly residues, which exists in animals and plants to defend against oxidative stress (Gu et al. 2012).

In this study, we chemically synthesized all of the tripeptides that could possibly be derived from $\beta-\mathrm{Lg}$ based on its amino acid sequence, and evaluated their antioxidant activities using a ferric-reducing antioxidant power (FRAP) assay. A QSAR model was constructed to develop a relationship between the antioxidant activity of $\beta-\mathrm{Lg}$ tripeptides and their structure.

\section{Materials and methods}

\subsection{Chemicals}

TPTZ (2,4,6-tripyridyl-s-triazine) and glutathione (GSH) were purchased from Sigma-Aldrich (St. Louis, MO, USA). $O$-(Benzotriazol-1-yl)- $N, N, N^{\prime}, N^{\prime}$ tetramethyluronium hexafluoro-phosphate (HBTU), 1-hydroxybenzotriazole (HOBT), thioanisole, N,N-diisopropylcarbodiimide (DIC), ethanedithiol (EDT), trifluoroacetic acid (TFA), diisopropylethylamine (DIPEA), and all Fmocprotected amino acids were purchased from GL Biochem (Shanghai, China). Wang resin and 2-chlorotrityl resin were purchased from NankaiHecheng Science and Technology Co. Ltd. (Tianjin, China). HPLC-grade methanol and acetonitrile were purchased from Fisher Scientific (Loughborough, UK). All of the other reagents used in the current study were obtained commercially as the analytical grade. 


\subsection{Peptide synthesis and purification}

$\beta$-Lg was selected as the parent protein in the current study, and the sequence of the bovine $\beta$-Lg precursor (Mercadante et al. 2012) is shown below:

\section{MKCLLLALAL TCGAQALIVT QTMKGLDIQK VAGTWYSLA 41 AASDISLLDA QSAPLRVYVE ELKPTPEGDL EILLQKWENG 81 ECAQKKIIAE KTKIPAVFKI DALNENKVLV LDTDYKKYLL 121 FCMENSAEPE QSLACQCLVR TPEVDDEALE KDKALKALP 161 MHIRLSFNPT QLEEQCHI}

This particular sequence provided 175 tripeptides, with each peptide having a two-amino-acid overlap. These 175 tripeptides contained three repeated sequences, which were removed to reduce the total number of tripeptides to 172 (Table 1).

All of the peptides were synthesized using solid phase Fmoc Chemistry (Ren et al. 2011). 2-Chlorotrityl resin was used for the peptide acids containing carboxyl-terminal Trp, Cys, and Pro residues, whereas Wang resin was used for all of the other peptide acids. When more than one tripeptide shared the same N-terminal sequence, resins bearing different pre-loaded C-terminal amino acids were mixed together, with up to four tripeptides being synthesized in one batch.

All of the individual peptides and peptide mixtures were purified using a Shimadzu (Kyoto, Japan) HPLC equipped with LC-10AT pumps and an SPD-10A VP Plus UV detector. HPLC purifications were conducted over an Agilent Eclipse XDB-C18 column $(9.4 \times 250 \mathrm{~mm}, 5 \mu \mathrm{m})$. The mobile phases used for the HPLC were $0.1 \%$ TFA in water (solvent A) and $0.1 \%$ TFA in acetonitrile (solvent B). The elution gradients were varied slightly depending on the peptide sequence. In a typical purification run, the solvent gradient was increased from 5 to $40 \%$ B in a linear manner over $40 \mathrm{~min}$ at a flow rate of $5 \mathrm{~mL} \cdot \mathrm{min}^{-1}$. The solvent eluting from the column was monitored at 230 (for those without Trp) or $280 \mathrm{~nm}$ (for those with Trp). The peptide purities were determined to be $>95 \%$ in all cases by RP-HPLC using a Kramsil C18 column (4.6× $250 \mathrm{~mm}$ ) and an elution gradient of 5 to $50 \% \mathrm{~B}$ over $30 \mathrm{~min}$ at a flow rate of $1 \mathrm{~mL} \cdot \mathrm{min}^{-1}$. All of the peptides were characterized by ESI-MS using an Agilent 1100 Series LC/ MSD (Santa Clara, CA, USA). The LC/MS system was operated under following conditions: HV capillary, 3,500 V; nebulizer pressure, 15 psi; dry gas flow rate, 5 L.min ${ }^{-1}$; and dry gas temperature, $325^{\circ} \mathrm{C}$.

\subsection{Ferric-reducing antioxidant power assay (FRAP assay)}

The FRAP assay was performed according to the method described by Benzie and Strain (1996) with some minor modifications. Ten microliters of a sample solution containing $100 \mathrm{mmol} . \mathrm{mL}^{-1}$ of peptides was incubated at $37^{\circ} \mathrm{C}$ with $100 \mu \mathrm{L}$ of FRAP reagent, containing $10 \mathrm{mmol} . \mathrm{L}^{-1}$ of TPTZ and $20 \mathrm{mmol}^{-1}$ of $\mathrm{FeCl}_{3}$. The absorption values were read after $10 \mathrm{~min}$ at a wavelength of $570 \mathrm{~nm}$ using a microplate reader (Model 680, Bio-Rad, Hercules, CA, USA). Aqueous $\mathrm{Fe}^{2+}$ solutions at concentrations in the range of $10-1,000 \mu \mathrm{mol} . \mathrm{L}^{-1}$ were used to produce a calibration curve. The activity of glutathione (GSH) was also measured as a positive control. The results were 
Table 1 Antioxidant activities of the 172 tripeptides based on $\beta$-Lactoglobulin

No. Tripeptide Relative activity No. Tripeptide Relative activity No. Tripeptide Relative activity sequence $\left(\mathrm{mmol} \mathrm{Fe}_{\mathrm{mol}}^{-1}\right) \quad$ sequence $\left(\mathrm{mmol} \mathrm{Fe}^{\left.-\mathrm{mol}^{-1}\right)}\right.$ sequence $\left(\mathrm{mmol} \mathrm{Fe}^{\left.-\mathrm{mol}^{-1}\right)}\right.$

\begin{tabular}{|c|c|c|c|c|c|c|c|c|}
\hline 1 & MKC & $5.25 \pm 0.13$ & 59 & EEL & $21.53 \pm 5.60$ & 117 & YLL & $81.76 \pm 6.27$ \\
\hline 2 & KCL & $11.19 \pm 0.09$ & 60 & ELK & $0.22 \pm 0.13$ & 118 & LLF & $1.05 \pm 0.12$ \\
\hline 3 & CLL & $7.74 \pm 1.66$ & 61 & LKP & $0.04 \pm 0.04$ & 119 & LFC & $117.66 \pm 4.31$ \\
\hline 4 & LLL & $0.83 \pm 0.15$ & 62 & KPT & $0.52 \pm 0.06$ & 120 & FCM & $32.5 \pm 0.83$ \\
\hline 5 & LLA & $0.20 \pm 0.03$ & 63 & PTP & $1.53 \pm 0.00$ & 121 & CME & $97.72 \pm 0.73$ \\
\hline 6 & LAL & $5.73 \pm 0.010$ & 64 & TPE & $0.26 \pm 0.07$ & 122 & MEN & $5.37 \pm 4.83$ \\
\hline 7 & ALA & $0.19 \pm 0.010$ & 65 & PEG & $0.19 \pm 0.04$ & 123 & ENS & $0.46 \pm 0.05$ \\
\hline 8 & ALT & $0.00 \pm 0.00$ & 66 & EGD & $0.14 \pm 0.04$ & 124 & NSA & $0.43 \pm 0.03$ \\
\hline 9 & LTC & $676.99 \pm 4.14$ & 67 & GDL & $0.00 \pm 0.00$ & 125 & SAE & $0.55 \pm 0.04$ \\
\hline 10 & TCG & $4.67 \pm 0.17$ & 68 & DLE & $0.06 \pm 0.05$ & 126 & AEP & $0.18 \pm 0.05$ \\
\hline 11 & CGA & $12.04 \pm 0.19$ & 69 & LEI & $1.46 \pm 0.60$ & 127 & EPE & $0.00 \pm 0.04$ \\
\hline 12 & GAQ & $0.37 \pm 0.02$ & 70 & EIL & $0.00 \pm 0.00$ & 128 & PEQ & $0.22 \pm 0.02$ \\
\hline 13 & AQA & $1.66 \pm 0.02$ & 71 & ILL & $0.35 \pm 0.01$ & 129 & EQS & $0.42 \pm 0.03$ \\
\hline 14 & QAL & $5.37 \pm 0.05$ & 72 & LLQ & $0.76 \pm 0.18$ & 130 & QSL & $0.32 \pm 0.10$ \\
\hline 15 & ALI & $0.17 \pm 0.08$ & 73 & LQK & $0.23 \pm 0.05$ & 131 & LAC & $48.89 \pm 2.54$ \\
\hline 16 & LIV & $0.14 \pm 0.08$ & 74 & QKW & $106.28 \pm 2.17$ & 132 & ACQ & $23.81 \pm 2.18$ \\
\hline 17 & IVT & $0.11 \pm 0.03$ & 75 & KWE & $8.01 \pm 0.10$ & 133 & CQC & $338.84 \pm 14.54$ \\
\hline 18 & VTQ & $0.11 \pm 0.17$ & 76 & WEN & $20.55 \pm 0.70$ & 134 & QCL & $49.38 \pm 2.44$ \\
\hline 19 & TQT & $1.81 \pm 0.03$ & 77 & ENG & $0.43 \pm 0.05$ & 135 & CLV & $114.14 \pm 0.78$ \\
\hline 20 & QTM & $6.31 \pm 0.10$ & 78 & NGE & $0.50 \pm 0.09$ & 136 & LVR & $1.35 \pm 0.01$ \\
\hline 21 & TMK & $4.38 \pm 0.10$ & 79 & GEC & $43.29 \pm 1.42$ & 137 & VRT & $0.35 \pm 0.63$ \\
\hline 22 & MKG & $6.35 \pm 0.44$ & 80 & ECA & $11.87 \pm 0.25$ & 138 & RTP & $0.26 \pm 0.37$ \\
\hline 23 & KGL & $0.00 \pm 0.02$ & 81 & CAQ & $13.26 \pm 0.28$ & 139 & PEV & $0.06 \pm 0.02$ \\
\hline 24 & GLD & $0.19 \pm 0.04$ & 82 & $\mathrm{AQK}$ & $0.15 \pm 0.05$ & 140 & EVD & $0.01 \pm 0.03$ \\
\hline 25 & LDI & $0.18 \pm 0.02$ & 83 & QKK & $0.14 \pm 0.04$ & 141 & VDD & $0.01 \pm 0.02$ \\
\hline 26 & DIQ & $0.50 \pm 0.00$ & 84 & KKI & $1.28 \pm 0.05$ & 142 & $\mathrm{DDE}$ & $0.13 \pm 0.01$ \\
\hline 27 & IQK & $0.00 \pm 0.00$ & 85 & KII & $0.00 \pm 0.00$ & 143 & DEA & $0.01 \pm 0.02$ \\
\hline 28 & QKV & $0.00 \pm 0.00$ & 86 & IIA & $0.15 \pm 0.01$ & 144 & EAL & $0.00 \pm 0.12$ \\
\hline 29 & KVA & $2.02 \pm 0.06$ & 87 & IAE & $0.32 \pm 0.02$ & 145 & ALE & $0.03 \pm 0.02$ \\
\hline 30 & VAG & $2.55 \pm 0.17$ & 88 & AEK & $0.11 \pm 0.04$ & 146 & LEK & $0.02 \pm 0.02$ \\
\hline 31 & AGT & $1.09 \pm 0.24$ & 89 & $\mathrm{EKT}$ & $0.42 \pm 0.02$ & 147 & $\mathrm{EKF}$ & $3.45 \pm 0.07$ \\
\hline 32 & GTW & $327.80 \pm 32.10$ & 90 & KTK & $0.31 \pm 0.57$ & 148 & KFD & $0.12 \pm 0.00$ \\
\hline 33 & TWY & $9.05 \pm 0.07$ & 91 & TKI & $0.08 \pm 0.01$ & 149 & FDK & $0.85 \pm 0.05$ \\
\hline 34 & WYS & $13.15 \pm 0.15$ & 92 & KIP & $0.07 \pm 0.02$ & 150 & DKA & $0.00 \pm 0.00$ \\
\hline 35 & YSL & $6.51 \pm 0.08$ & 93 & IPA & $0.14 \pm 0.02$ & 151 & KAL & $0.00 \pm 0.06$ \\
\hline 36 & SLA & $4.42 \pm 0.52$ & 94 & PAV & $1.10 \pm 0.02$ & 152 & ALK & $0.17 \pm 0.00$ \\
\hline 37 & LAM & $7.07 \pm 0.58$ & 95 & AVF & $2.28 \pm 0.06$ & 153 & LKA & $0.00 \pm 0.00$ \\
\hline 38 & AMA & $0.39 \pm 0.02$ & 96 & VFK & $0.17 \pm 0.04$ & 154 & ALP & $2.33 \pm 0.09$ \\
\hline 39 & MAA & $2.76 \pm 0.67$ & 97 & FKI & $0.23 \pm 0.00$ & 155 & LPM & $10.99 \pm 0.43$ \\
\hline 40 & AAS & $0.23 \pm 0.08$ & 98 & KID & $0.39 \pm 0.13$ & 156 & PMH & $0.99 \pm 0.03$ \\
\hline
\end{tabular}


Table 1 (continued)

\begin{tabular}{|c|c|c|c|c|c|c|c|c|}
\hline No. & $\begin{array}{l}\text { Tripeptide } \\
\text { sequence }\end{array}$ & $\begin{array}{l}\text { Relative activity } \\
\left(\mathrm{mmol} \mathrm{Fe} \cdot \mathrm{mol}^{-1}\right)\end{array}$ & No. & $\begin{array}{l}\text { Tripeptide } \\
\text { sequence }\end{array}$ & 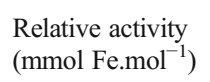 & No. & $\begin{array}{l}\text { Tripeptide } \\
\text { sequence }\end{array}$ & $\begin{array}{l}\text { Relative activity } \\
\left(\mathrm{mmol} \mathrm{Fe} \cdot \mathrm{mol}^{-1}\right)\end{array}$ \\
\hline 41 & ASD & $0.30 \pm 0.03$ & 99 & IDA & $0.21 \pm 0.02$ & 157 & MHI & $14.47 \pm 0.45$ \\
\hline 42 & SDI & $0.13 \pm 0.02$ & 100 & DAL & $0.01 \pm 0.01$ & 158 & HIR & $1.31 \pm 0.12$ \\
\hline 43 & DIS & $0.19 \pm 0.07$ & 101 & ALN & $1.51 \pm 0.04$ & 159 & IRL & $0.24 \pm 0.02$ \\
\hline 44 & ISL & $0.22 \pm 0.04$ & 102 & LNE & $0.57 \pm 0.11$ & 160 & RLS & $1.10 \pm 0.14$ \\
\hline 45 & SLL & $1.15 \pm 0.01$ & 103 & NEN & $11.94 \pm 0.84$ & 161 & LSF & $4.87 \pm 0.71$ \\
\hline 46 & LLD & $0.06 \pm 0.04$ & 104 & ENK & $0.27 \pm 0.09$ & 162 & SFN & $1.17 \pm 0.05$ \\
\hline 47 & LDA & $0.17 \pm 0.00$ & 105 & NKV & $0.00 \pm 0.00$ & 163 & FNP & $0.63 \pm 0.12$ \\
\hline 48 & DAQ & $0.46 \pm 0.02$ & 106 & KVL & $0.12 \pm 0.05$ & 164 & NPT & $0.67 \pm 0.07$ \\
\hline 49 & AQS & $0.27 \pm 0.07$ & 107 & VLV & $3.36 \pm 0.25$ & 165 & PTQ & $2.75 \pm 0.81$ \\
\hline 50 & QSA & $0.47 \pm 0.03$ & 108 & LVL & $0.95 \pm 0.12$ & 166 & TQL & $0.03 \pm 0.02$ \\
\hline 51 & SAP & $0.83 \pm 0.04$ & 109 & VLD & $0.24 \pm 0.03$ & 167 & QLE & $0.90 \pm 0.17$ \\
\hline 52 & APL & $0.30 \pm 0.02$ & 110 & LDT & $4.20 \pm 0.17$ & 168 & LEE & $0.03 \pm 0.01$ \\
\hline 53 & PLR & $0.36 \pm 0.38$ & 111 & DTD & $0.00 \pm 0.00$ & 169 & EEQ & $0.97 \pm 0.03$ \\
\hline 54 & LRV & $1.57 \pm 0.13$ & 112 & TDY & $10.33 \pm 0.16$ & 170 & EQC & $33.33 \pm 1.29$ \\
\hline 55 & RVY & $8.92 \pm 0.08$ & 113 & DYK & $11.46 \pm 0.12$ & 171 & $\mathrm{QCH}$ & $9.99 \pm 1.35$ \\
\hline 56 & VYV & $15.38 \pm 0.09$ & 114 & YKK & $1.79 \pm 0.05$ & 172 & $\mathrm{CHI}$ & $28.07 \pm 1.15$ \\
\hline 57 & YVE & $11.17 \pm 0.00$ & 115 & KKY & $11.97 \pm 0.12$ & & & \\
\hline 58 & VEE & $0.13 \pm 0.04$ & 116 & KYL & $12.16 \pm 0.33$ & & & \\
\hline
\end{tabular}

The activities have been reported as the averages of three independent experiments \pm standard deviation. The bold regions represent the peptides with antioxidant activities greater than $100 \mathrm{mmol} \mathrm{Fe}^{3+} \cdot \mathrm{mol}^{-1}$ peptide. The activity of glutathione $\left(273.28 \pm 12.13 \mathrm{mmol} \mathrm{Fe} 3+. \mathrm{mol}^{-1}\right.$ peptide) was measured as a positive control

expressed as micromoles $\mathrm{Fe}^{2+}$ equivalents per mole of the sample based on a standard curve of $\mathrm{FeSO}_{4} \cdot 7 \mathrm{H}_{2} \mathrm{O}$. All of these experiments were carried out in triplicate.

\subsection{QSAR modeling}

\subsubsection{Structural characterization and regression analysis}

The antioxidant activities of the 172 peptides synthesized in the current study were subjected to a data standardization protocol to make up the dependent variables. The divided physicochemical property score (DPPS) descriptors (Tian et al. 2009) were applied to describe the tripeptides, which make up the independent variables. The DPPS descriptors were made up of 10 variables which were used to represent the electronic $(X 1-4)$, steric $(X 5-6)$, hydrophobic $(X 7-8)$, and hydrogen-bonding properties $(X 9-10)$ of each individual amino acid. The amino acids in the sequence from $\mathrm{N}$ terminal to the C-terminal were designed as $n, m$, and $c$, respectively. Subsequently, each tripeptides had 30 descriptors (i.e., $3 \times 10$ ), which were expressed as $n X 1-10$, $m X 1-10$, and $c X 1-10$. The relationships between the biological activity and the peptide descriptor variable matrix were then modeled by multiple linear regression (MLR) analysis using the version R2013a of the MATLAB software (The MathWorks, Inc., Natick, MA, USA). 


\subsubsection{Model evaluation and cross validation}

Before modeling, five peptides with the highest activities between which there were large gaps (that exceed $10-15 \%$ of the entire range of activities) and 14 inactive peptides were suppressed. The peptides left were then randomly divided into training and test sets in a 2:1 ratio. The training dataset was used to establish the QSAR models and perform an internal validation process, whereas the test set was used to perform an external validation process. The quality of the fit between the model-predicted and experimental values, and the suitability of the regression model in terms of its ability to describe the data were measured by the coefficient of determination $\left(R^{2}\right)$ of the training set and Fisher's test value ( $F$ value). The actual stability of the QSAR model was measured by $Q^{2}$ (the leave-one-out cross-validation $R^{2}$ ) and the $R_{\text {test }}^{2}$ of the test dataset. The robustness was also measured by the randomization of the modeled property (Yscrambling).

\section{Results and discussions}

\subsection{The antioxidant activity of tripeptides}

The antioxidant activities of the 172 synthetic tripeptides were evaluated using a FRAP assay, with GSH being used as a positive control. The activities observed in a FRAP assay are presented as the amount of $\mathrm{Fe}^{3+}$ reduced per mole of antioxidant, which allows for a direct comparison between results generated under different testing concentrations. The relative activities of the 172 tripeptide and GSH were calculated, and the results are listed in Table 1.

As glutathione, the well-known multifunctional antioxidant, shows activity around $273 \mathrm{mmol} \mathrm{Fe}^{3+} \cdot \mathrm{mol}^{-1}$ peptide, we consider the range around its activity as "high," that is to say, above $100 \mathrm{mmol} \mathrm{Fe}{ }^{3+} \cdot \mathrm{mol}^{-1}$ peptide. Marcuse (1960) found that some of the amino acids can act as primary antioxidants themselves; we assessed the activity of some free amino acids and found that Trp and Cys have activities around 5.96 and $50.74 \mathrm{mmol} \mathrm{Fe}^{3+} \cdot \mathrm{mol}^{-1}$ amino acid. Therefore, we consider $1-100 \mathrm{mmol} \mathrm{Fe}^{3+} \cdot \mathrm{mol}^{-1}$ peptide as "moderate," and lower than $1 \mathrm{mmol} \mathrm{Fe}^{3+} \cdot \mathrm{mol}^{-1}$ peptide as "low." More than half of the peptides showed very poor antioxidant activities at a concentration of $100 \mathrm{mmol} . \mathrm{L}^{-1}$. Seventy-four of the peptides showed antioxidant activities greater than $1 \mathrm{mmol} \mathrm{Fe}^{3+} \cdot \mathrm{mol}^{-1}$ peptide. Among this particular group of tripeptides, six peptides (LTC, CQC, GTW, LFC, CLV, and QKW) showed high activities, and three peptides, LTC, CQC, and GTW, showed higher activities than that of the GSH.

\subsection{Model evaluation and cross validation}

As shown in Table 2, the values of $R^{2}$ and $F$ were 0.643 and 7.324, respectively, which suggested that the activities of these peptides shared a linear relationship with the physicochemical properties of the individual residues in their sequence at $5 \%$ significance level. Figure 1a shows a plot of the calculated activities against the observed activities for the training set. The relationship between the observed and predicted activities of the test set is shown in Fig. 1b. The scatter plots of the estimates 
Table 2 Statistical parameters of the MLR model for the QSAR of the 172 antioxidative tripeptides based on $\beta$-Lactoglobulin

\begin{tabular}{lrlll}
\hline Statistics & $n^{\mathrm{a}}$ & $R^{2 \mathrm{~b}}$ & $Q^{2 \mathrm{c}}$ & $F^{\mathrm{d}}$ \\
\hline Training set & 108 & 0.643 & 0.541 & 7.324 \\
Test set & 46 & 0.635 & - & - \\
\hline
\end{tabular}

\footnotetext{
${ }^{a}$ Number of samples

${ }^{\mathrm{b}}$ Coefficient of determination

${ }^{\mathrm{c}}$ Cross-validation $R^{2}$

${ }^{\mathrm{d}} F$ value in $F$ test
}

vs the experimental values are rather evenly distributed along the regression line, as well for the training set than for the test set, suggesting a reliability of the model.

The external validation of the current model was performed using the prediction dataset. The $Q^{2}$ and $R_{\text {test }}^{2}$ values were 0.541 and 0.635 , respectively, which suggested that the model was creditable and stable. The model was further validated by applying the Y-randomization test. Nineteen random shuffles of the Y-vector gave $R^{2}$ values in the ranges of $0.143-0.368$ and $Q^{2}$ values in the ranges of $0.000-0.208$ as shown in Table 3. The confidence level is $95 \%$. The low $R^{2}$ and $Q^{2}$ values obtained show that the good results in our original model are not due to a chance correlation or structural dependency of the training set.

\subsection{Relationship between antioxidant activity and amino acids in the peptide sequences}

Figure 2 shows the regression coefficients of the different variables for the tripeptides. These regression coefficients revealed that $m X 9, c X 6, m X 10, c X 1, n X 10, c X 10, c X 4$, $n X 6, m X 2$, and $n X 1$ made a much greater contribution than any of the other variables. Furthermore, $n X 8, n X 9, m X 9, c X 5$, and $\mathrm{c} X 8$ had a negative effect on the antioxidant activity; whereas, all of the other variables had a positive impact. Based on the meaning of the DPPS descriptors and the positions of different amino acids in the sequences, it was clear the electronic and hydrogen-bonding properties of the amino acids in the tripeptide sequences, as well as the steric properties of the amino acid residues at the Cand N-termini, played an important role in the antioxidant activities of the tripeptides. Considering that the dependent variables (i.e., antioxidant activities) obtained were presented in a reducing capacity based on their performance in the FRAP assay, which provides a direct measurement of the antioxidant activity through reduction reaction based on single electron transfer, it is perhaps not surprising that the results of the regression showed that the antioxidant activity was mainly related to the electronic and hydrogen-bonding properties of the amino acid residues.

Based on the regression equation from the QSAR modeling, we calculated the antioxidant activities of tripeptides bearing one of the 20 common amino acids at their $\mathrm{C}$ - or N-terminus, or in the middle of their sequence in an attempt to predict which peptide sequence would provide the strongest activity. It turned out to be a similar rank 

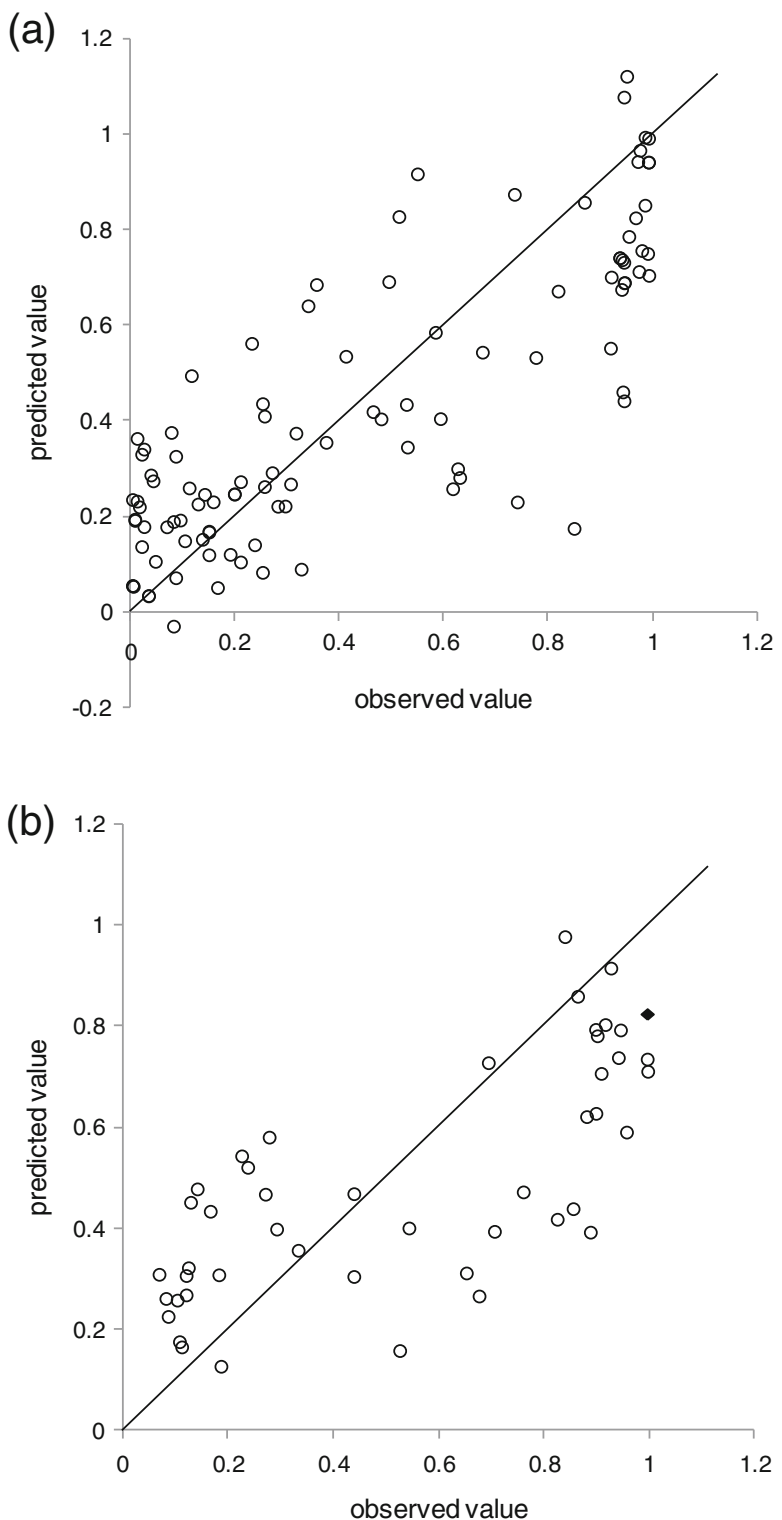

Fig. 1 Calculated antioxidant values versus observed values a for the training set and $\mathbf{b}$ for the test set. The circles represent the chemosynthetic tripeptides, whereas the diamond represents the GSH. Outliers were excluded before the modeling process for the training set

for the predicted values, with Cys (C) and Trp (W) residues tending to provide higher predicted values.

\subsection{Illustration of the structural characteristics of antioxidative peptides}

Numerous studies have been reported in the literature that peptides containing Cys (C) and Trp (W) amino acid residues have higher antioxidant activities than peptides not 
Table 3 Y-randomization results of the QSAR model of the 172 antioxidative tripeptides based on $\beta$ Lactoglobulin
${ }^{\mathrm{a} C o e f f i c i e n t}$ of determination

${ }^{\mathrm{b}}$ Cross-validation $R^{2}$

\begin{tabular}{lll}
\hline No. & $R^{2 \mathrm{a}}$ & $Q^{2 \mathrm{~b}}$ \\
\hline 1 & 0.279 & 0.061 \\
2 & 0.277 & 0.000 \\
3 & 0.223 & 0.012 \\
4 & 0.310 & 0.100 \\
5 & 0.282 & 0.069 \\
6 & 0.323 & 0.113 \\
7 & 0.343 & 0.146 \\
8 & 0.340 & 0.129 \\
9 & 0.329 & 0.117 \\
10 & 0.340 & 0.123 \\
11 & 0.337 & 0.000 \\
12 & 0.346 & 0.019 \\
13 & 0.368 & 0.208 \\
14 & 0.235 & 0.019 \\
15 & 0.264 & 0.044 \\
16 & 0.260 & 0.011 \\
17 & 0.143 & 0.000 \\
18 & 0.246 & 0.000 \\
19 & 0.356 & 0.176 \\
\hline
\end{tabular}

containing these residues. An early study indicated that the antioxidant effect of $\beta$-Lg in the raw milk was associated with the free sulfhydryl group at Cys-121 (Liu et al. 2007). Saito et al. (2003) found that tripeptides containing Trp (W) at the C-terminus had strong radical scavenging ability. Other oligopeptides with high antioxidant activity were also separated and identified containing one or more Trp (W) from $\beta$-Lg (Contreras et al. 2011; Hernández-Ledesma et al. 2005), $\beta-C N$ (Suetsuna et al. 2000), and sardinelle (Bougatef et al. 2010).

For a given amino acid or peptide to act as an antioxidant, it must be preferentially oxidized in the presence of other compounds in the food matrix. In principle, all 20 of the amino acids found in proteins could interact with free radicals if the energy of the

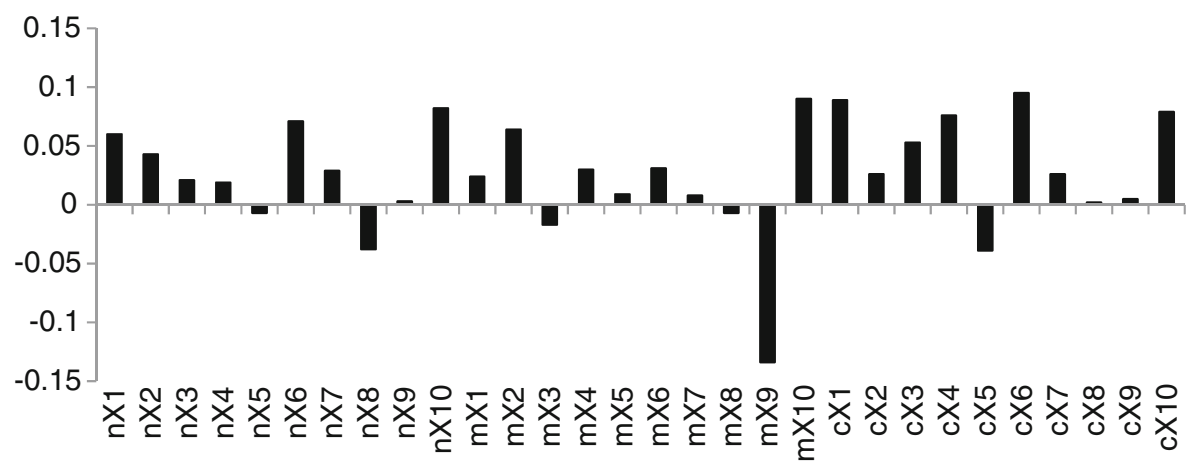

Fig. 2 Plot of regression coefficients for the 172 tripeptides based on $\beta$-Lactoglobulin 
free radical was high enough (Samaranayaka and Li-Chan 2011). The oxidative reactivities of a variety of amino acids were evaluated by photochemical oxidation with an $\mathrm{OH}$-containing compound and it was found that the reactivities of the amino acids were of the order Cys $>$ Trp and Tyr $>$ Met $>$ Phe $>$ His $>$ Ile $>$ Leu $>$ Pro (Sharp et al. 2004). The sulfhydryl (R-SH) group in Cys (C) has a unique antioxidant activity and interacts with radical species by hydrogen donation from the $\mathrm{SH}$ group or the loss of an electron from its sulfur atom (Elias et al. 2008). Residues with an aromatic ring structure (i.e., Trp W) have been reported to donate a proton to electron deficient radicals (Chen et al. 1998). Taken together, these results demonstrate that the nucleophilic sulfur-containing amino acids Cys (C) and the aromatic amino acids Trp (W) make a significant contribution to the antioxidant activities of certain peptide.

This result appeared to contradict the results of previous studies ( $\mathrm{Li}$ and Li 2013; Li et al. 2011) in some cases where it may prefer different amino acids at different positions of the sequence, although these differences in the results were mainly due to the differences in the assay conditions. The FRAP assay measures the antioxidant activity through a reduction reaction based on single electron transfer reactions, where the redox potential of the compounds is important. Whereas the ORAC, SOR, and TEAC assays are based on a free radical scavenging system which are influenced quite strongly by the type of reactive species used. Although TEAC assays is also based on single electron transfer reactions and the redox potential of the redox couple, ABTS/ABTS ${ }^{+}(0.68 \mathrm{~V})$, is comparable to that of $\mathrm{Fe}(\mathrm{II}) /(\mathrm{III})(0.70 \mathrm{~V})$, the TEAC and FRAP assays provided different results because the two assays operate under different reaction conditions, including different $\mathrm{pH}$ values (e.g., FRAP, $\mathrm{pH}$ 3.6; TEAC, $\mathrm{pH}$ 7.4) and different levels of steric hindrance for the molecules being oxidized (Müller et al. 2011). Assays carried out in the presence of lipids (e.g., FTC assay) or an emulsion to inhibit lipid peroxidation could correlate more closely with the amphiphilic and steric properties of the antioxidants, which would help them enter into the lipid system so that they could react with the free radicals.

It is interesting to note that despite peptides with Cys (C) or Trp (W) in the sequence show higher activities, their activities still vary. This may be attributed to the influence of neighboring amino acids beside these two particular amino acid residues in the peptide sequence. The strong radical scavenging ability of $\operatorname{Trp}(\mathrm{W})$ residues may be explained by the special capability of indolic group to serve as hydrogen donor and the stability of the indolic radical which depends on neighboring amino acid residues in the presence of an acid-ferric ion system (Saito et al. 2003). That is to say, the physicochemical properties of neighboring amino acids may influence the ability to donate electron of the Cys or Trp, and thus, influence the antioxidant activity. However, a further study is still needed to understand the influence of neighboring amino acid residues and its mechanism.

\section{Conclusions}

In summary, all the tripeptides that could possibly derive from bovine betalactoglobulin have been synthesized, and their antioxidant activities measured using a ferric-reducing antioxidant power assay. The results revealed that the antioxidant activities of three tripeptides, LTC, CQC, and GTW, were higher than that of GSH. 
A QSAR model of antioxidant activities of these peptides was also performed. The results of QSAR modeling showed that the electronic and hydrogen-bonding properties of the amino acids in the tripeptide sequences, as well as the steric properties of the amino acid residues at the $\mathrm{C}$ - and $\mathrm{N}$-termini, played an important role in the antioxidant activities of the tripeptides. Tripeptides exhibiting the strongest antioxidant activities tended to contain Cys (C) and Trp (W) residues.

Acknowledgments This work is financially supported by the Ministry of Science and Technology of China (2012BAD12B08), and the International Science \& Technology Cooperation Program of China (2011DFA32550).

Declaration of conflicting interests The Authors declare that there is no conflict of interest.

\section{References}

Benzie IFF, Strain JJ (1996) The ferric reducing ability of plasma (FRAP) as a measure of "antioxidant power": the FRAP assay. Anal Biochem 239(1):70-76

Bougatef A, Nedjar-Arroume N, Manni L, Ravallec R, Barkia A, Guillochon D, Nasri M (2010) Purification and identification of novel antioxidant peptides from enzymatic hydrolysates of sardinelle (Sardinella aurita) by-products proteins. Food Chem 118(3):559-565

Chen HM, Muramoto K, Yamauchi F, Nokihara K (1996) Antioxidant activity of designed peptides based on the antioxidative peptide isolated from digests of a soybean protein. J Agric Food Chem 44(9):2619-2623

Chen HM, Muramoto K, Yamauchi F, Fujimoto K, Nokihara K (1998) Antioxidative properties of histidinecontaining peptides designed from peptide fragments found in the digests of a soybean protein. J Agric Food Chem 46(1):49-53

Contreras MM, Hernández-Ledesma B, Amigo L, Martín-Álvarez PJ, Recio I (2011) Production of antioxidant hydrolyzates from a whey protein concentrate with thermolysin: optimization by response surface methodology. LWT-Food Sci Technol 44(1):9-15

Elias RJ, Kellerby SS, Decker EA (2008) Antioxidant activity of proteins and peptides. Crit Rev Food Sci Nutr 48(5):430-441

Gu Y, Wu J (2013) LC-MS/MS coupled with QSAR modeling in characterising of angiotensin I-converting enzyme inhibitory peptides from soybean proteins. Food Chem 141(3):2682-2690

Gu L, Zhao M, Li W, You L, Wang J, Wang H, Ren J (2012) Chemical and cellular antioxidant activity of two novel peptides designed based on glutathione structure. Food Chem Toxicol 50(11):4085-4091

Hernández-Ledesma B, Dávalos A, Bartolomé B, Amigo L (2005) Preparation of antioxidant enzymatic hydrolysates from $\alpha$-lactalbumin and $\beta$-lactoglobulin. Identification of active peptides by HPLC-MS/MS. J Agric Food Chem 53(3):588-593

Hernandez-Ledesma B, Recio I, Amigo L (2008) beta-Lactoglobulin as source of bioactive peptides. Amino Acids 35(2):257-265

Jenssen H, Fjell CD, Cherkasov A, Hancock RE (2008) QSAR modeling and computer-aided design of antimicrobial peptides. J Pept Sci 14(1):110-114

Jian-Hui W, Yong-Le L, Jing-Heng N, Jian Y, Xiang-Hong L, Fa-Xiang W (2013) Is the structural diversity of tripeptides sufficient for developing functional food additives with satisfactory multiple bioactivities? J Mol Struct 1040:164-170

Jing P, Qian B, He Y, Zhao X, Zhang J, Zhao D, Lv Y, Deng Y (2014) Screening milk-derived antihypertensive peptides using quantitative structure activity relationship (QSAR) modelling and in vitro/in vivo studies on their bioactivity. Int Dairy J 35(1):95-101

Korhonen H (2009) Milk-derived bioactive peptides: from science to applications. J Funct Foods 1(2):177187

Li YW, Li B (2013) Characterization of structure-antioxidant activity relationship of peptides in free radical systems using QSAR models: key sequence positions and their amino acid properties. J Theor Biol 318 : $29-43$ 
Li YW, Li B, He J, Qian P (2011) Structure-activity relationship study of antioxidative peptides by QSAR modeling: the amino acid next to C-terminus affects the activity. J Pept Sci 17(6):454-462

Liu H, Chen W, Mao S (2007) Antioxidant nature of bovine milk $\beta$-Lactoglobulin. J Dairy Sci 90(2):547-555

Marcuse R (1960) Antioxidative effect of amino-acids. Nature 186(4728):886-887

Mercadante D, Melton LD, Norris GE, Loo TS, Williams MA, Dobson RC, Jameson GB (2012) Bovine $\beta$ Lactoglobulin is dimeric under imitative physiological conditions: dissociation equilibrium and rate constants over the $\mathrm{pH}$ range of 2.5-7.5. Biophys J 103(2):303-312

Moller NP, Scholz-Ahrens KE, Roos N, Schrezenmeir J (2008) Bioactive peptides and proteins from foods: indication for health effects. Eur J Nutr 47(4):171-182

Müller L, Fröhlich K, Böhm V (2011) Comparative antioxidant activities of carotenoids measured by ferric reducing antioxidant power (FRAP), ABTS bleaching assay ( $\alpha$ TEAC), DPPH assay and peroxyl radical scavenging assay. Food Chem 129(1):139-148

Ren F, Zhang S, Guo H, Jiang L (2011) Systemic screening of milk protein-derived ACE inhibitors through a chemically synthesised tripeptide library. Food Chem 128(3):761-768

Saito K, Jin D-H, Ogawa T, Muramoto K, Hatakeyama E, Yasuhara T, Nokihara K (2003) Antioxidative properties of tripeptide libraries prepared by the combinatorial chemistry. J Agric Food Chem 51(12): 3668-3674

Sakai A, Miyata N, Takahashi A (1997) Promoting activity of 3-tert-butyl-4-hydroxyanisole (BHA) in BALB/3 T3 cell transformation. Cancer Lett 115(2):213-220

Samaranayaka AGP, Li-Chan ECY (2011) Food-derived peptidic antioxidants: a review of their production, assessment, and potential applications. J Funct Foods 3(4):229-254

Sharp JS, Becker JM, Hettich RL (2004) Analysis of protein solvent accessible surfaces by photochemical oxidation and mass spectrometry. Anal Chem 76(3):672-683

Suetsuna K, Ukeda H, Ochi H (2000) Isolation and characterization of free radical scavenging activities peptides derived from casein. J Nutr Biochem 11(3):128-131

Tian F, Yang L, Lv F, Yang Q, Zhou P (2009) In silico quantitative prediction of peptides binding affinity to human MHC molecule: an intuitive quantitative structure-activity relationship approach. Amino Acids 36(3):535-554

Wu J, Aluko RE, Nakai S (2006a) Structural requirements of angiotensin I-converting enzyme inhibitory peptides: quantitative structure-activity relationship modeling of peptides containing 4-10 amino acid residues. QSAR Comb Sci 25(10):873-880

Wu J, Aluko RE, Nakai S (2006b) Structural requirements of angiotensin I-converting enzyme inhibitory peptides: quantitative structure - activity relationship study of di- and tripeptides. J Agric Food Chem 54(3):732-738

Yan L, Yan Y, Liu H, Lv Q (2013) Stepwise identification of potent antimicrobial peptides from human genome. Biosystems 113(1):1-8 